\title{
Basic Development Stages of the Algorithms Applied to Recover Lost Microacceleration Data and Check Efficiency of Measuring Equipment on the Board of Space Laboratory
}

\section{K. I. Potienko}

Faculty of Computer Information Technologies and Automatic Equipment, Student of Donetsk National Technical University, Donetsk, Ukraine

\section{Email address: \\ potienko97@mail.ru}

\section{To cite this article:}

K. I. Potienko. Basic Development Stages of the Algorithms Applied to Recover Lost Microacceleration Data and Check Efficiency of Measuring Equipment on the Board of Space Laboratory. American Journal of Aerospace Engineering. Special Issue: Space Laboratories: History, Researches, Prospects. Vol. 3, No. 1-1, 2015, pp. 10-16. doi: 10.11648/j.ajae.s.2016030101.13

Abstract: The following article deals with the algorithm applied to recover lost microacceleration data. The algorithm allows recovering on the basis of available part of measuring data. It is based on the fractal quality of constructive part of microacceleration field. The algorithm can be applied for technological space laboratories only if a constructive part of microacceleration field is prevalent.

Keywords: Space Laboratory, Algorithm Applied to Recover Data, Microaccelerations

\section{Introduction}

Microacceleration level in the working area of technological equipment is one of the most important characteristics of modern space laboratory [1]. The problem to measure microaccelerations during orbital flight of space laboratory correctly is very difficult. The causes of it are mentioned below:

1. Highly sensitive equipment can fail because of great overloads which can achieve $10 \mathrm{~g}$ during launching of unmanned space laboratory.

For example, during different sets of experiments of Microgravity Isolation Mount (MGIM) in the board of space station "MIR" amplitudes of oscillations differed in 20 times $[1,2]$. The data of microaccelerations which were measured by French microaccelerometers "BETA" on the board of uncontrolled and unmanned space laboratory "Foton-11" which are shown in figure 1 were similar [3]. Readings of accelerometers for three different axes of a bound coordinate system differed also in 20 times.

2. There are some difficulties to measure microaccelerations directly in contrast to temperature, pressure etc. That is why different gauges give different data, especially if these gauges are based on different methods to estimate microaccelerations and have different sensitivity. Readings of French equipment "Alice-2" and Perm Convection Sensor "DACON" on the board can exemplify it $[1,4]$.

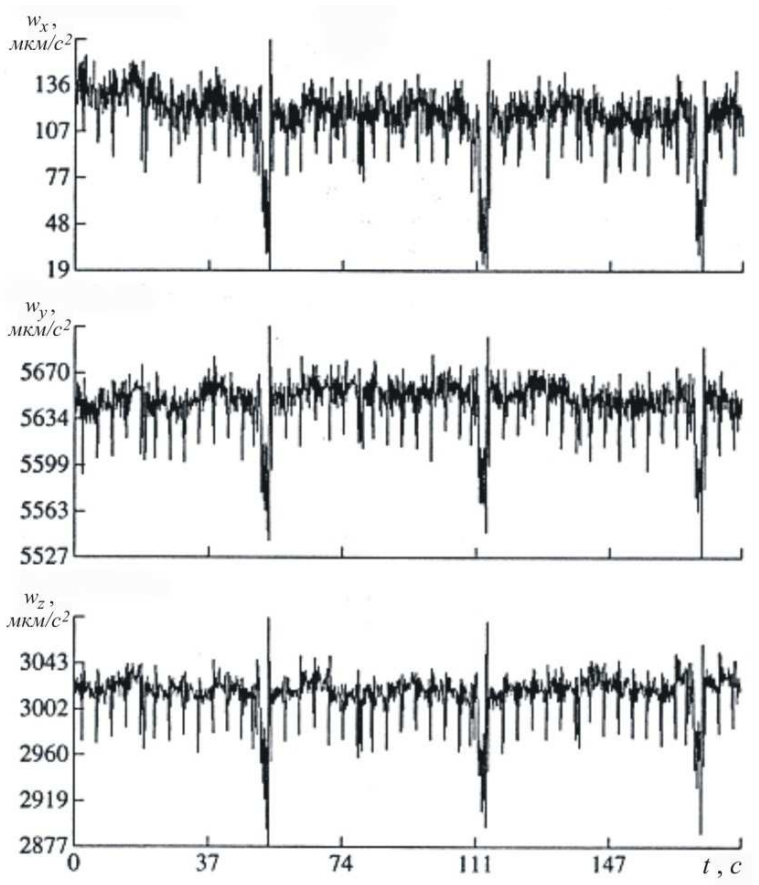

Figure 1. The estimation of microaccelerations on the board of space laboratory "Foton-11" by French microaccelerometers "BETA".

Valid data are necessary to explore how microacceleration 
field effects on gravity-sensitive processes. Some articles [5-7] show ambiguity of this effect by the example of oriented crystallization. That is why the problem to increase data reliability is of great urgency for development of space technologies and space laboratories.

\section{Microaccelerometers}

\subsection{Classification of Microaccelerometers}

Microaccelerometers can be categorized on the basis of their operating principle. There are three groups of ones: electric, mechanical and electromechanical.

Operating principle of electric accelerometers (figure 2) is based on changing of electro-capacity between static and dynamic states.

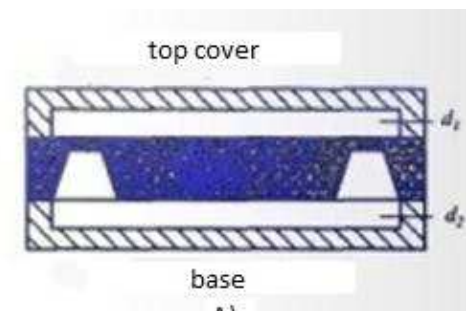

A)

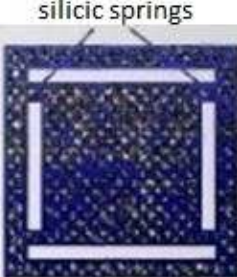

B)
Figure 2. Capacitive accelerometer with differential capacitor: A) cross-section of accelerometer;B) top view..

Thereafter a capacitor must be included in construction of the detector. Measuring of microscopical amplitudes of vibration oscillations requires high-accuracy detector of such movements. Maximum movement which can be measured by electric accelerometers is about $20 \mu \mathrm{m}$. This condition significantly effect on range of application of these accelerometers. Electric microaccelerometers are used for measuring of low-frequency vibrations, movement and constant accelerations. The range of application of these gauges is very comprehensive. However, there is a significant disadvantage: electrostatic forces changes running of accelerometer. So these gauges hardly can be tested on the Earth [8].

Mechanical microaccelerometers (figure 3) significantly differ from electric ones. The basis constructional part of mechanical detector is a pendulum. The sensor records its displacement from point of equilibrium. Each pendulum accelerometer has elastic suspension, which compensate a torque caused by microaccelerations. Elastic suspension moves pendulum because they are connected. The construction of accelerometer also should involve some element, which recover pendulum, such as return induction coil. Thus, microaccelerations can be estimated on the basis of certain displacement of elastic suspension caused by small forces. However, presence of elastic suspension in construction of accelerometer puts on certain application conditions and application sphere [9].

Recently developments, which include pendulum accelerometers as well as capacitive, have appeared. Sensor of these accelerometers is made from monocrystalline silicon and glass.

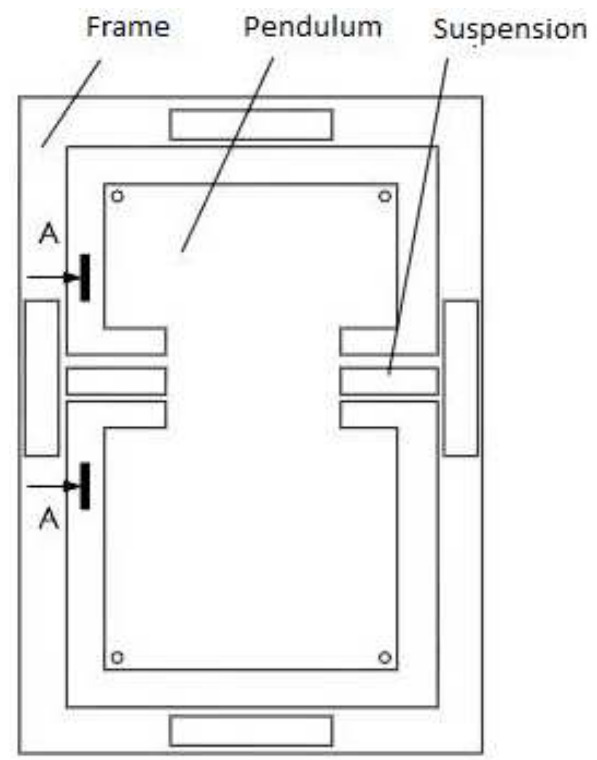

Figure 3. Space laboratoty “Foton-M" No 3.

It guarantees high reliability, accuracy, time and temperature stability of the gauges. If the sensor has measurement range about $\pm 1 \mathrm{~g}$, detector can withstand loads about $50000 \mathrm{~g}$. At the same time it is sensitive to static acceleration and vibration. Moreover, important criteria of accelerometers is its measuremenrt, frequency, temperature range, maximum shock load, number of axes, access speed, size and mass. For example, great overloads have their action on space laboratory during its going to orbit. Usually, low-sensitive capacitive accelerometers can support such overloads. These gauges run on the board of space laboratory until it has gone to its orbit. High-accuracy pendulum accelerometers are running during orbital flight. So microaccelerometers based on microelectromechanical system are the most practically feasible.

\subsection{Examples}

Microgravity Acceleration Measurement System (MAMS) studies the small forces, or vibrations and accelerations, on the International Space Station (ISS) that result from the operation of hardware, crew activities, dockings and maneuvering. Results are used to generalize the types of vibrations affecting vibration-sensitive experiments. Investigators seek to better understand the vibration environment on the space station. Vibrations exist on the ISS from a variety of sources, such as equipment operation, life-support systems, crew activities, aerodynamic drag, gravity gradient, rotational effects and the vehicle structural resonance frequencies. The quasi-steady acceleration is caused by forces from aerodynamic drag, gravity gradient effects, centripetal (rotational) motion, spacecraft propulsion, and vehicle orientation control actions. MAMS consists of two sensors, the Orbital Acceleration Research Experiment (OARE) Sensor Subsystem (OSS) and the High Resolution Accelerometer Package (HiRAP), monitor these disturbances. The OARE OSS measures low 
range frequency (up to $1 \mathrm{~Hz}$ ). The HiRAP characterizes the ISS vibratory environment from $0.01 \mathrm{~Hz}$ to $100 \mathrm{~Hz}$. These quasi-steady state accelerations occur in the frequency range below $1 \mathrm{~Hz}$. MAMS consists of a low-frequency triaxial accelerometer, the Miniature Electro-Static Accelerometer (MESA), a high-frequency accelerometer, the High-Resolution Accelerometer Package (HiRAP), and associated computer, power, and signal processing subsystems contained within a Double Middeck Locker enclosure.

The DIMAC (Direct-measurement Microaccelerometer) system developed for the "Foton-M" № 3 flight is a modular inertial monitoring system which measures vibrations, quasi-steady accelerations and the Earth magnetic field. It was built at RedShift Design and Engineering BVBA of Sint-Niklaas, Belgium.

The DIMAC system consists of DIMAC Control Unit, DIMAC Sensor Head and three DIMAC Magnetometer Modules (figure 4).

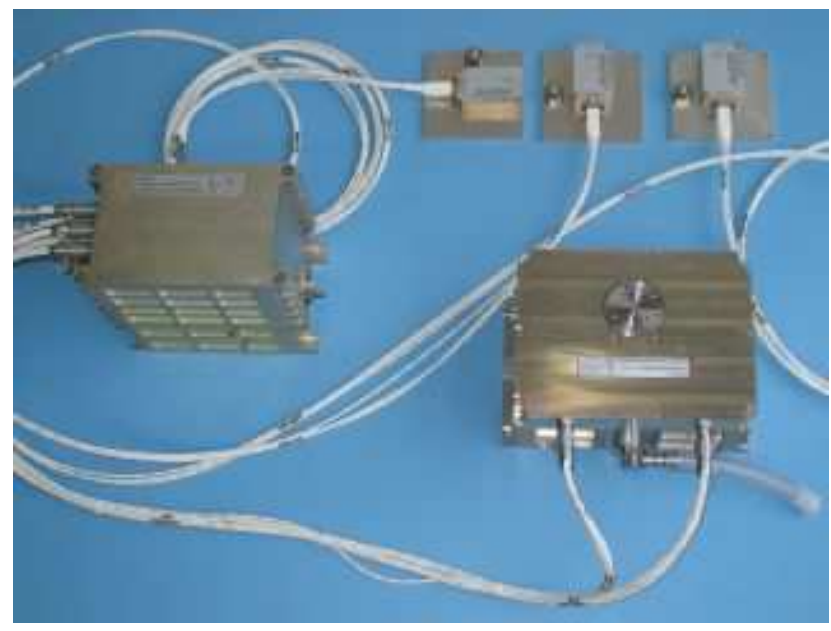

Figure 4. Overview of DIMAC.

The DIMAC Control Unit is an enhanced version of the TAS3 device featuring: autonomous acquisition of internal tri-axial vibration sensor (based on Honeywell QA3000 series sensors), acquisition of vibration data (resolution $1 \mu \mathrm{g}$, bandwidth $0.01 \mathrm{~Hz}$ to $200 \mathrm{~Hz}$, sample rate $1000 \mathrm{~Hz}$ ) and sensor temperature data. The DIMAC Sensor Head is a proto-flight version of the experimental quasi-steady acceleration sensor developed during the DIMAC study phase. For the "Foton-M" №3 mission the DIMAC Sensor Head was located at the bottom of the re-entry capsule, close to the center of gravity of the spacecraft. This sensor was implemented in order to correct for the minor magnetic field susceptibility of the acceleration measurements that was detected during the processing of TAS3 measurement data after the "Foton-M" №2 mission.

Tri-axial magnetometer modules are measuring the Earth magnetic field (and hence indirectly the spacecraft attitude). Three modules have been placed at different locations inside the "Foton-M" №3 re-entry capsule in order to compensate for any local magnetic field disturbances caused by payloads or spacecraft subsystems.
The stored data have been downloaded, post-processed and analysed after the mission. During the mission subsets of the DIMAC measurement data have been downloaded using the telemetry interface. These data have been processed immediately and preliminary results have been available to the scientists and operators of other "Foton-M" №3 payloads. The flexible implementation of the telecommand interface allowed telemetry data bandwidth optimisation and DIMAC system troubleshooting. However, the flight results show no major disturbances. This indicates that if an appropriate location is selected a single magnetometer module might be sufficient for future missions. The modularity of the DIMAC system allows easy implementation of additional sensor modules. The resulting system could provide full inertial and environmental monitoring of a spacecraft for future missions. The major parts of this system have been qualified on the "Foton-M" №3 mission.

The majority of microgravity experiments show their highest sensitivity to residual acceleration in the low frequency range, typically $<0.01 \mathrm{~Hz}$, where atmospheric drag, gravity gradient and centrifugal forces are pre-dominant. QSAM (Quasi-Steady Accelerometer Measurement System) is an instrument especially developed to detect this range where conventional methods are hampered by bias and noise problems. Bias is generally varying in time due to unknown dependence on temperature, ageing and other effects precluding pre-mission calibration. In QSAM, signal modulation is applied to cancel the instantaneous bias during the measurement cycle. The modulation is achieved by turning the sensor's sensitive axis. QSAM is capable to detect the frequency range between 0 and $0.02 \mathrm{~Hz}$ with a resolution better than $10^{-7} \mathrm{~g}_{0}$.

STAR (Space Three-axis Accelerometer for Research mission) which is shown in figure 5 is an accelerometer system provided by CNES and developed by ONERA in France.

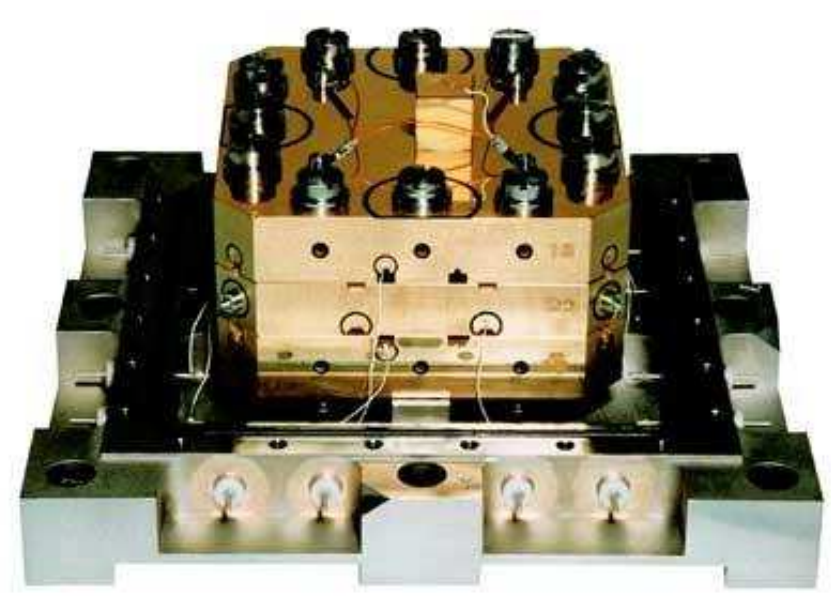

Figure 5. Overview of STAR.

The objective is to measure all non-gravitational accelerations of the satellite (drag, solar and Earth radiation pressure) in order to determine the Earth's gravity field from purely gravitational orbit perturbations (orbit from BlackJack). 
The accelerometer measurement principle is based on electrostatic suspension of a proof-mass in a cage. Instantaneous position of the proof-mass is measured by three capacitive sensors which permit a determination of the acceleration vector. The instrument has a dynamic range of $\pm 10^{-4} \mathrm{~ms}^{-2}$, a resolution of better than $\pm 3 \cdot 10^{-9} \mathrm{~ms}^{-2}$, and a frequency range of $10^{-1}$ to $10^{-4} \mathrm{~Hz}$. The STAR instrument is positioned at the center of gravity of CHAMP to minimize the influence of measurement disturbances due to rotational accelerations and gravity gradients. STAR is also connected to a star sensor (ASC of DTU, Denmark) with two heads to provide the accelerometer's axes orientation. The accelerometer proof-mass is positioned at the center of gravity of CHAMP.

Convection sensor DAKON-M is a cavity cylinder filled with carbon dioxide. Diameter and height of cylinder have the same value $\mathrm{L}=10 \mathrm{~cm}$. The set temperature difference $\Delta \mathrm{T}=60^{\circ} \mathrm{C}$ is kept fixed on the opposite ends of the sensor. There are two differential thermocouples to measure temperature differences in the two pairs of fixed points inside the cavity. The sensor shows these differences. Convection sensor DAKON-M is an improved version of DAKON sensor, which was tested on the orbital station "MIR" [1]. According to [2-5] data from the sensor depend on microaccelerations experienced by it. This sensor is an example of a gravitationally sensitive system.

It is worth to say that on the orbital station "MIR" was tested French accelerometer Alice-2 along with DAKON (figure 6). However, the data of two different sensors were different.

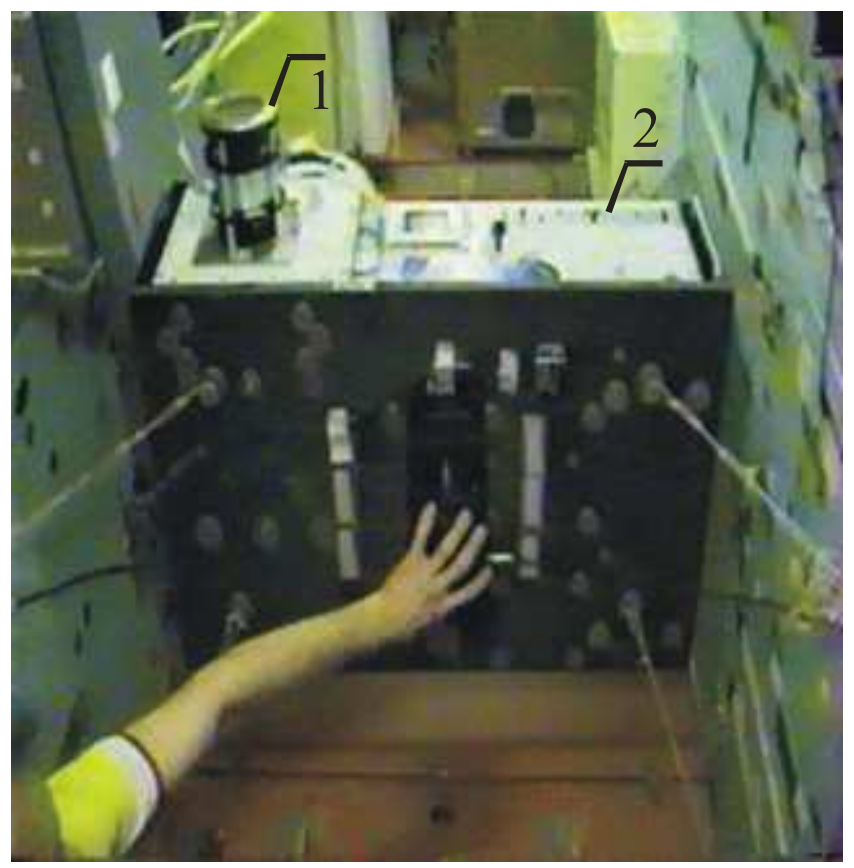

Figure 6. Convection sensor DAKON (1) and equipment Alice-2 (2).

During the last experiments on the board of "Foton-M" №4 was applied equipment to measure microaccelerations "GRAVITON" which is shown in figure 7. Its measurements are based on analyze of effect of microaccelerations on terrestrial magnetic field.

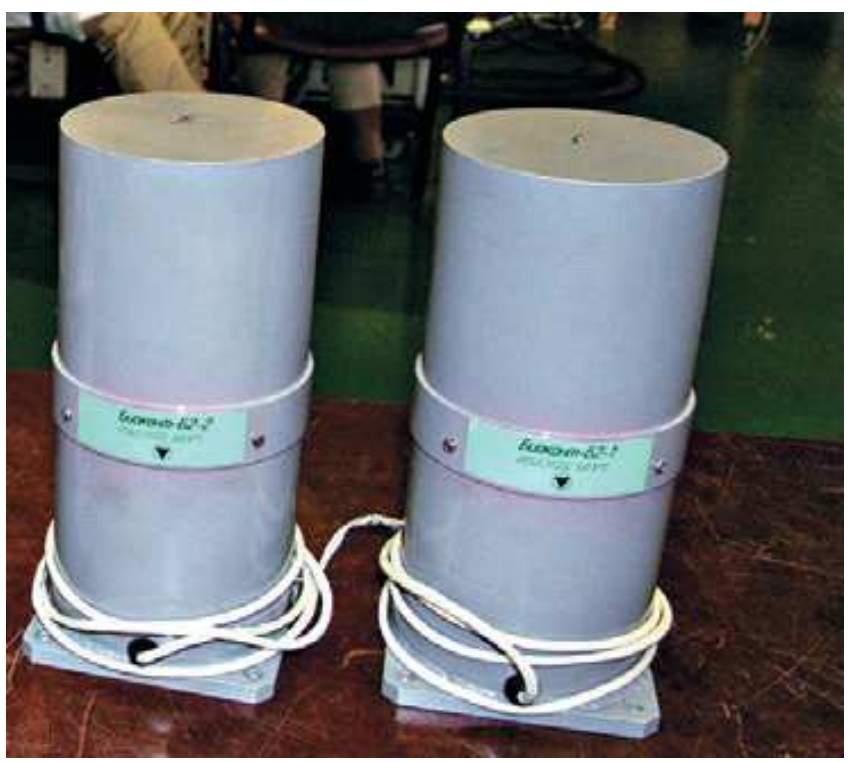

Figure 7. Overview of "GRAVITON".

Thus, accelerometers based on the different operational principle gives different data. It is one of the main problems how to measure accelerometers correctly.

\section{Fractal Quality of Constructive Microaccelerations}

Classification of microaccelerations according to ways of its control is shown in the article [1]. It divides all disturbing factors into three categories:

-metastable (outside disturbing factors and running of technological equipment);

-random (random oscillations of disturbing factors, micrometeorites, etc.);

-constructive (inside disturbing factors).

In some cases constructive microaccelerations mainly contribute to microacceleration field. Base model of such cases can be oriented orbital motion of space laboratory with big flexible elements [10]. Fractal (scaling) quality of microaccelerations was opened for similar cases [11]. It consists in invariability of microaccelerations dependence on time during scaling of sizes and inertia-mass characteristics of space laboratory. Method applied to recover lost microacceleration data was developed on the basis of this quality [12].

The purpose of the following article is to develop algorithms on the basis of the method [12].

\section{Development of Algorithms for Different Problems}

\subsection{The Problem to Recover Lost Part of Data}

Description: A part of data was lost during exploitation of 
space laboratory because of failure of gauges or problems with transmission of telemetric data.

Urgency: Solution of the problem will allow recovering of lost data on the basis of available part of data. This make possible to analyze facilities for realization of technological processes during all operational term. For instance, there was no communication with spacecraft from 19.07 to 26.07.2014 during realization of the project "Foton-M" №4. This circumstance caused some difficulties with telemetric data processing.

Algorithm:

1. Check availability of fractal quality by means of estimation of contribution of constructive part to microacceleration field.

2. Check possibility of recovering with prescribed accuracy by comparison of lost part with available part of data.

3. Estimate constructive part of microaccelerations from available part of data using fractal quality.

4. Check accuracy of estimation by comparison of model data with the available part.

5. Recover lost part of data using generated model.

This algorithm is shown in figure 8 .

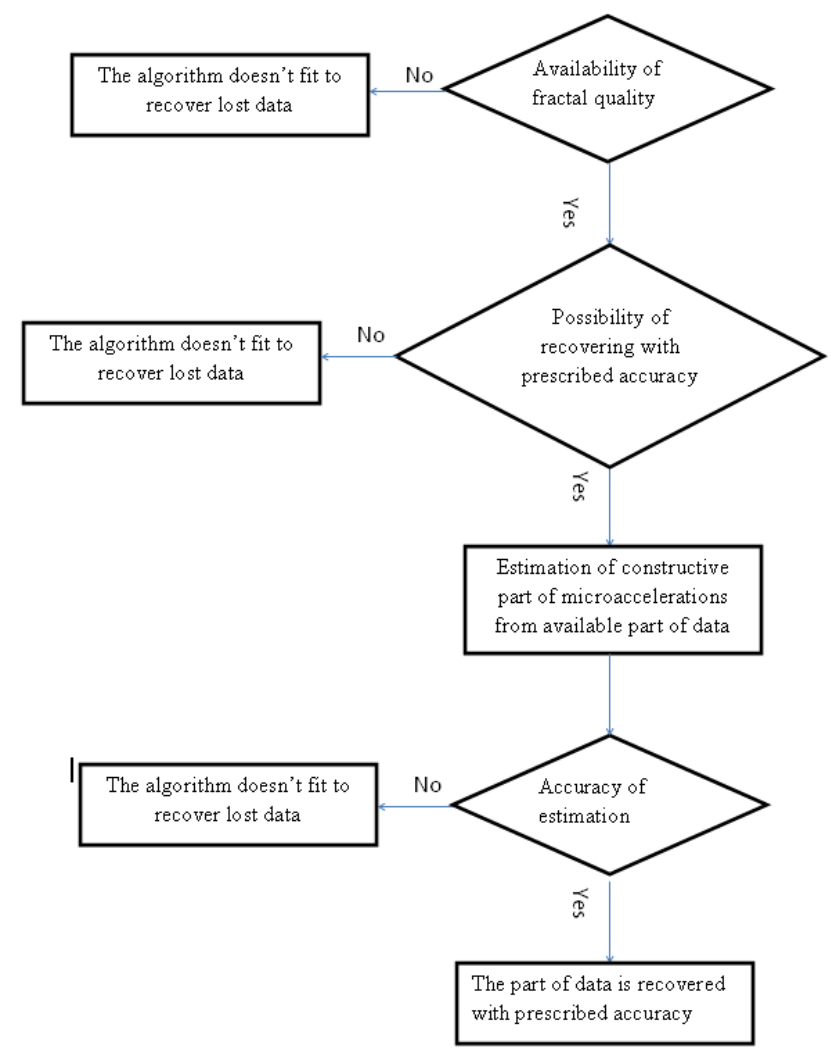

Figure 8. Algorithm 4.1.

\subsection{Correction of Data from Defective Gauges}

Description: Great launching overloads caused some gauges to fail during exploitation or software which is assigned to processing of information from gauges was running incorrectly.

Urgency: The practice of realization of space projects shows that in some cases gauges runs incorrectly. In figure are showed data of French accelerometers "BETA" for space laboratory "Foton-11". There were no physical cases for such significant differences of data from gauges [13]. That is why false run of gauges can be considered to be one of the possible cases. Incorrect data on the state of microacceleration field in the indoor environment of space laboratory doesn't allow analyzing of effect of microaccelerations on experiment. Requirements for successful realization of this experiment also will be wrong. Thus, the problem solution will allow:

-increasing of data accuracy;

-correcting of data from damaged gauges;

-analyzing of effect of microaccelerations on experimental results.

Algorithm:

1. Uncover significant differences of data from gages by test of statistic hypothesis of homogeneity.

2. Test availability of fractal quality by means of estimation of contribution of constructive part to microacceleration field.

3. Gather source data from each gauge to control them.

4. Estimate constructive part of microaccelerations on the basis of data from each gauge using fractal quality.

5. Generate secondary data in the form of deflection of source data from estimation of microaccelerations to control each gauge.

6. Analyze compliance between estimation of microaccelerations and source data by means of test of statistic hypothesis by fitting criterion.

7. Uncover damaged gauges.

8. Replace data by fractal estimation for each damaged gauge.

This algorithm is shown in figure 9.

\subsection{Efficiency Check of Gauges}

Description: It is necessary to control technological state of gauges on the stage of test and exploitation [14]. The control can be done by the compliance test of source data with fractal quality of microaccelerations.

Urgency: Efficiency check of gauges raises reliability of data. It makes possible to do passive control of gauges on the stage of test and exploitation. Moreover disadvantages of gauges can be detected by the efficiency check for the purpose of its development.

Algorithm:

1. Check availability of fractal quality by means of estimation of contribution of constructive part to microacceleration field.

2. Gather source data from gauges to control them.

3. Estimate constructional part of micriaccelerations using fractal quality.

4. Generate secondary data in the form of deflection of source data from estimation of microaccelerations.

5. Analyze compliance between estimation of microaccelerations and source data by means of test of statistic hypothesis by fitting criterion.

6. Draw a conclusion on correctness of data and technological state of gauges. 
This algorithm is shown in figure 10.

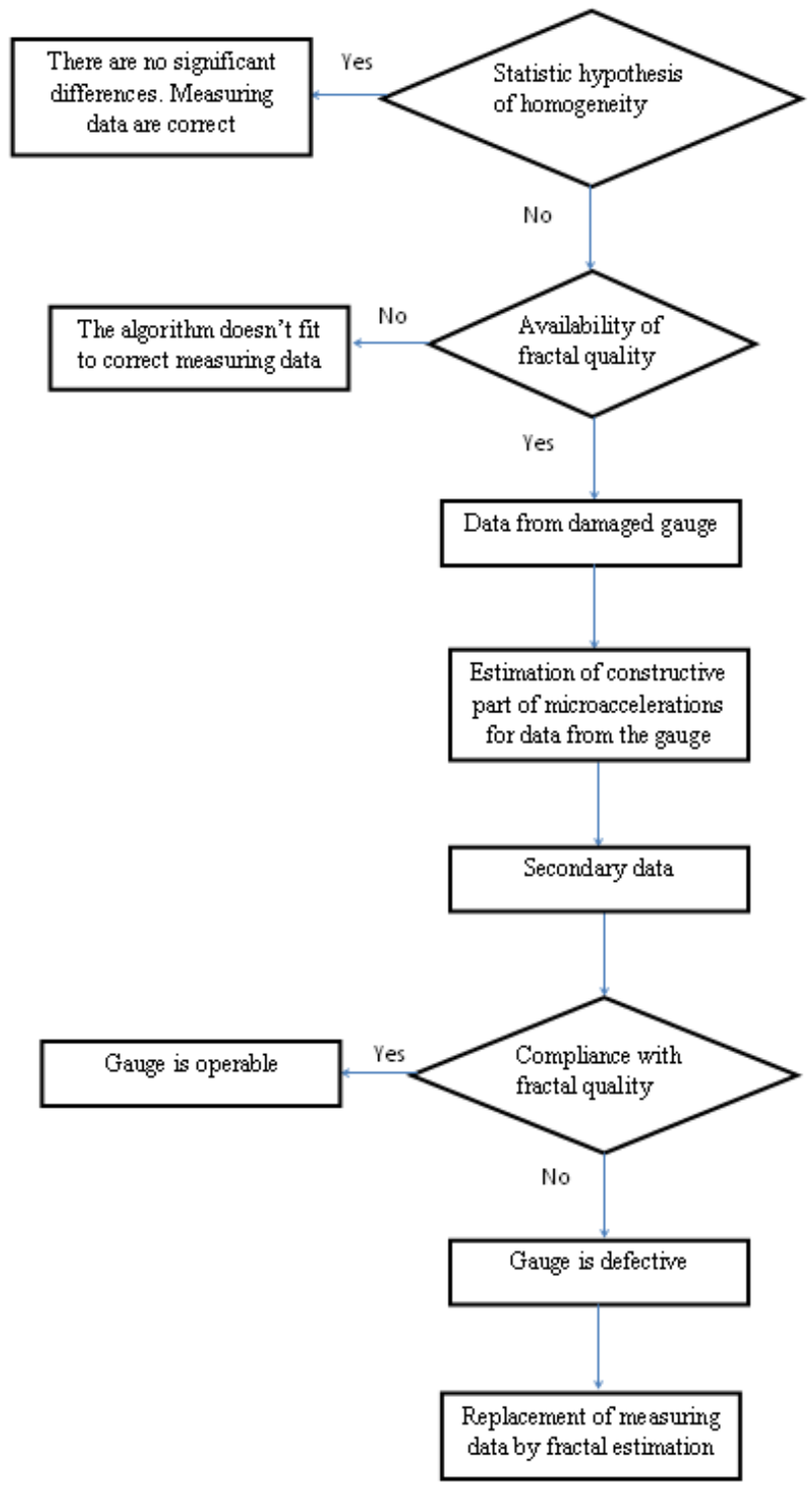

Figure 9. Algorithm 4.2.

\subsection{The Problem to Improve Accuracy of Data in Case of False Run of Gauges}

Description: False run of one or few gauges was uncovered by efficiency control of measuring equipment. It necessitates improvements of accuracy of data from the time period.

Urgency: In case some gauge are defective it is necessary to raise data accuracy to analyze facilities for realization of technological processes, uncover gravity-sensitive characteristics of this processes and also investigate effect of different factors on microacceleration field in the indoor environment of space laboratory.

Algorithm: Majority inspection [15] method is usually applied to raise accuracy of data in case of on or few gauges are damaged. This method is based on creating of additional measuring line by approximation of measured data. To algorithm to solute problem 3.3 precede solution of this problem. After this it is necessary to:

1. Estimate median of data on the basis of their measurement.

2. Make functional dependence.

3. Check compliancy between accuracy of approximation and prescribed accuracy.

4. Apply generated model as measuring data of all defective gauges.

This algorithm is shown in figure 11.

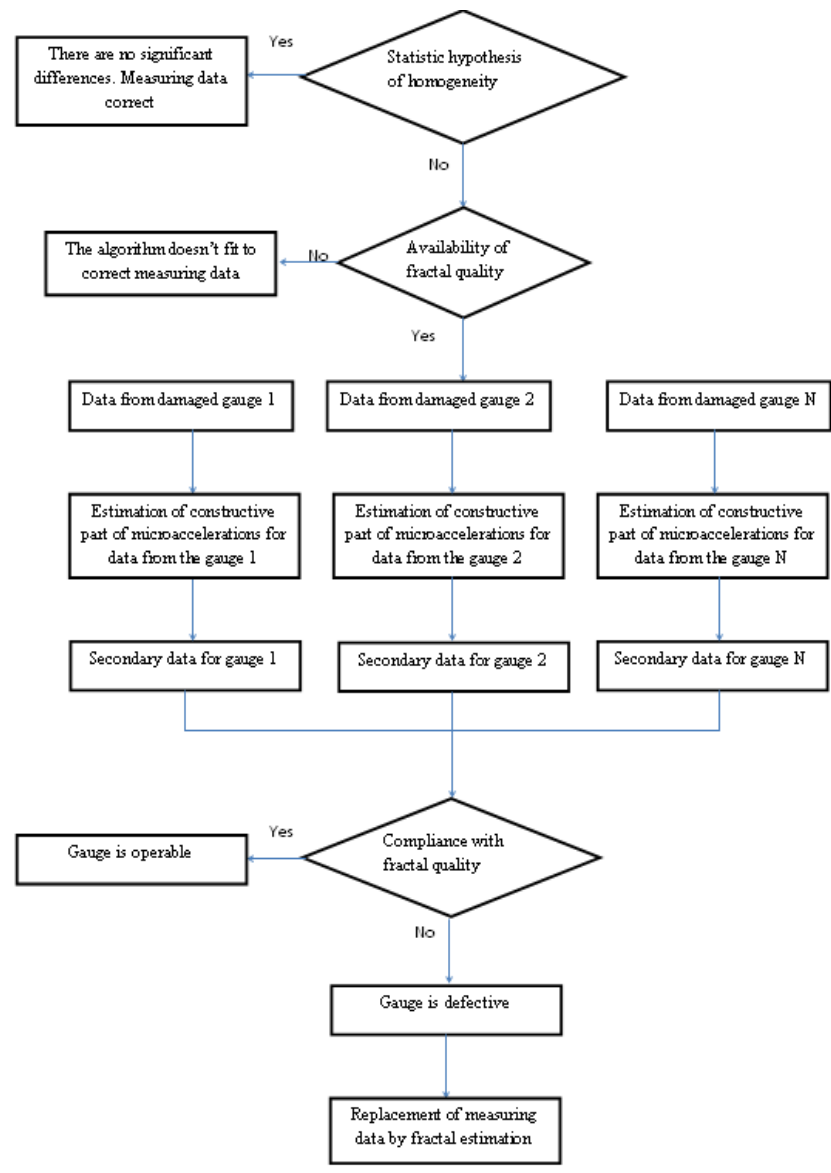

Figure 10. Algorithm 4.3.

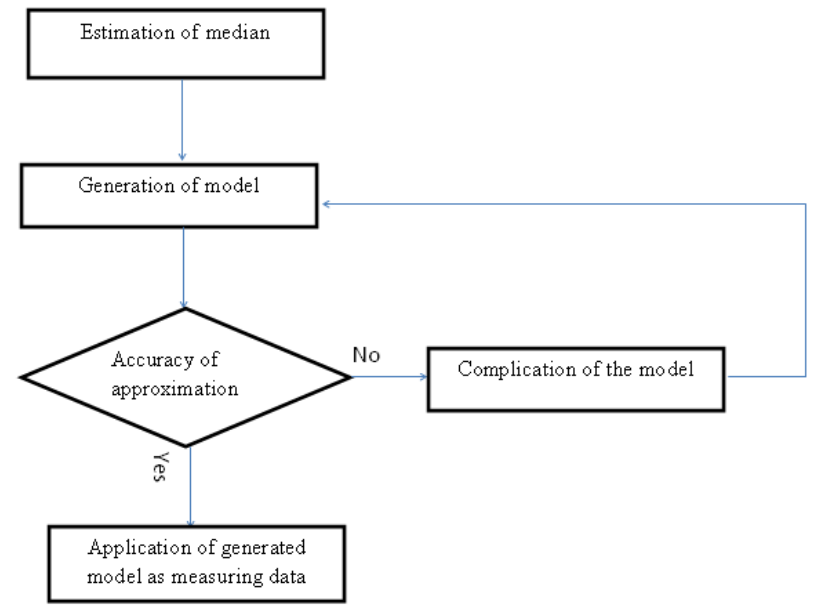

Figure 11. Algorithm 4.4. 


\section{Conclusion}

In the article are shown four different problems. They are concerned with recovering of lost measuring data, efficiency check of gauges and improvement of accuracy of measurements in case some gauges are damaged.

Reliable data are necessary for qualitative analyses of microacceleration field in the indoor environment of space laboratory.

If constructive part of microaccelerations is dominant the fractal quality of this part should be applied for effective solution of these problems. In case data comply with fractal quality they can be used for estimation of microaccelerations. Otherwise accuracy of measuring data can be improved by majority inspection method. Moreover the data can be replaced by fractal model.

The problems and algorithms which are shown in the article can be applied either for development of new space laboratories and gauges or for realization of space projects to conduct gravity-sensitive processes in space.

\section{References}

[1] A.V. Sedelnikov The Problem of Microaccelerations: from Comprehension up to Fractal Model, (Moscow, Russian Academy of Sciences: The Elected Works of the Russian School, 2012), p. 277.

[2] R.G. Owen, D.I. Jones, A.R. Owens and A.A. Robinson "Integration of a microgravity isolation mount within a Columbus single rack," Acta Astronautica, vol. 22, 1990, pp. 127-135.

[3] V.V. Sazonov, S. Yu. Chebukov, V.I. Abrashkin, A.E. Kazakova and A.S. Zaytsev "The analysis of low-frequency microaccelerations onboard FOTON-11 artificial satellite," Space Research, vol. 39, No 4, 2001, pp. 419-435.

[4] S.A. Nikitin, V.I. Polezhayev and V.V. Sazonov "About measurement quasistatic microacceleration components onboard an artificial satellite by means of the convection sensor," Space Research, vol. 39, No 2, 2001, pp. 179-187.

[5] V.S. Zemskov, M.R. Raukhman and V.P. Shalimov "Gravitational sensitivity of fusions at cultivation of crystals of InSb:Te Bridzhmen's by methods and bestigelny zonal melting in the conditions of a microgravity," Space Research, vol. 39, No 4, 2001, pp. 375-383.
[6] V.S. Zemskov, M.R. Raukhman and E.A. "Kozitsyna Features of crystallization of multicomponent alloys in the conditions of zero gravity," Physics and chemistry of processing of materials, No 5, 1985, pp. 44-49.

[7] V.S. Zemskov, M.R. Raukhman and V.P. Shalimov On the way to understanding of processes of cultivation from fusions of crystals of semiconductors in zero gravity on spacecrafts (Moscow, Institute of metallurgy and materials science of A.A. Baykov of 60 years, ELIS, Editions Lyakishev N. P., 1998), pp. 295-317.

[8] S.F. Konovalov, Yu.A. Ponomarev, D.V. Maiorov, V.P. Podchezercev and A.G. Sidorov "Hybrid microelectromechanical gyroscopes and acceleration gages," Science and Education, No 10, 2011, pp. 1-23.

[9] A.N. Boiko, A.V. Zavodyan and B.M. Simonov "Micromechanical accelerometers: modeling of elements of a design and production," Electronics: Science, Technology, Business, No 8, 2009, p.100-103.

[10] A.V. Sedelnikov and A.A. Kireeva "Alternative solutions to increase the duration of microgravity calm period on board the space laboratory," Acta Astronautica, vol. 69, 2011, pp. 480-484.

[11] A.V. Sedelnikov "Fractal quality of microaccelerations," Microgravity Scienes and Technology, vol. 24, No 5, 2012, pp. 345-350.

[12] A.V. Sedelnikov "The usage of fractal quality for microacceleration data recovery and for measuring equipment efficiency check," Microgravity Scienes and Technology, vol. 26, No 5, 2014, pp.327-334.

[13] A.I. Belousov and A.V. Sedelnikov "Probabilistic estimation of fulfilling favorable conditions to realize the gravity-sensitive processes aboard a space laboratory," Russian Aeronautics, vol. 56, No 3, 2013, p. 297-302.

[14] A.I. Belousov and A.V. Sedelnikov "Problems in formation and control of a required microacceleration level at spacecraft design, tests, and operation," Russian Aeronautics, vol. 57, No 2, 2014, pp. 111-117.

[15] S.V. Zhernakov "Algorithms of control and diagnostics of aviation gas-turbine engine in the conditions of onboard realization on the basis of technology of neural networks," The Bulletin of the Ufa State Aviation Technical University, vol. 14, No 3(38), 2010, pp. 42-56. 